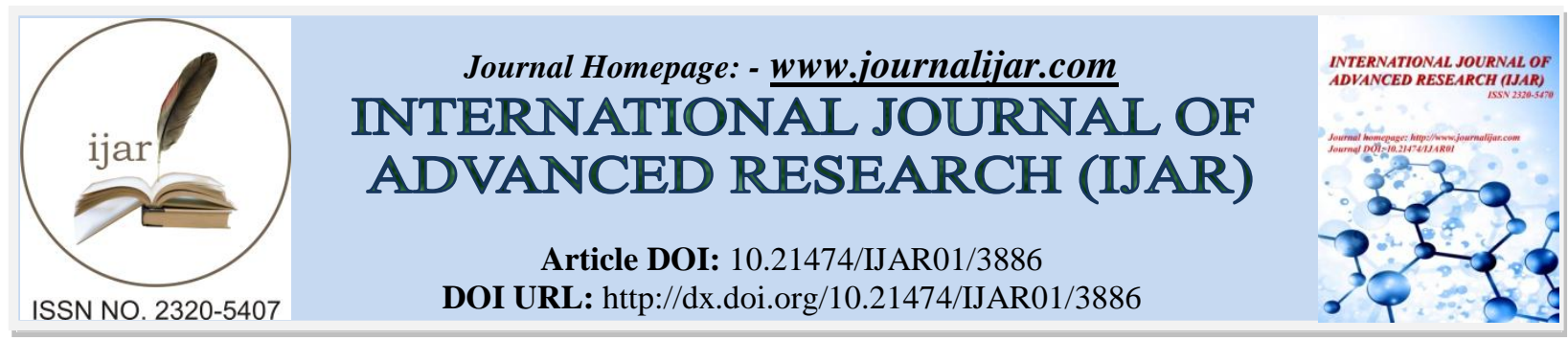

RESEARCH ARTICLE

\title{
A RELATIVE SURVEY OF VARIOUS ROUTING PROTOCOLS IN WSN.
}

\author{
Amit Kumar and Vishnu Sharma
}

CSE, Galgotias University.

\section{Manuscript Info}

Manuscript History

Received: 01 February 2017

Final Accepted: 01 March 2017

Published: April 2017

Key words:-

routing protocols, energy utilization, sensor hub arrangement, WSN, scalability, remote sensor.

\begin{abstract}
Advancement of WSN in ongoing applications, remote sensor system is most recent research field in Computer Network. WSN comprise of little, self-sufficient ease, control sensor hubs with detecting and remote correspondence capacities. The diagram of routing protocols for WSN is affected by testing elements as if energy utilization, adaptability, adaptation to noncritical failure, nature of administration. This survey paper contains, a comparative review based on aggregation of data, scalability and Quality of Service (QoS) for various routing protocols of Wireless Sensor Network. These arrangement of various routing protocols examined on the basis of: position based protocols, information driven protocols, in system and layered handling protocols, portability based protocols as well as information driven protocols.
\end{abstract}

Copy Right, IJAR, 2017,. All rights reserved.

\section{Introduction:-}

WSN is considered as a standout amongst the most imperative innovation in this day and age. Sensor system is empowered with advances remote correspondence innovation and installed with MEMS (microelectronic mechanical system). Small sensor hubs are easily sent and deployed in geographical territory and connected through remote connections. A remote sensor organize comprises of minimal effort, control, self-configurable and multifunctional sensor hubs conveyed in area of concern. At the point when contrasted and customary remote system, sensor networks have taking after attributes: thick hub arrangement, battery controlled sensor hubs, extreme energy and capacity requirement and successive topology change. Application of Wireless sensor organize secured numerous ranges as: ecological observing, war zone checking, human services applications, security and reconnaissance. A sensor hub comprises of four fundamental segments: control unit, detecting unit, handling unit and correspondence unit. The power unit is made up of battery for providing energy to function every single other segment in each hub design. These detecting components comprises of sensors as simple into advanced digitized converting device. The preparing unit comprises of and microcontroller/chip memory which give control to sensor hub. Thus, the sensors produce simple signals in light of execution of system. The correspondence unit comprises of wireless frequencies for executing transmission of information and as well as information gathering across the path or frequency. Accordingly, sensor structure assembled with minimal utilization of the power used by each sensor hub and as well as minimal price [1].

In this paper, a relative review of various routing protocols characterization is talked about, afterward examination is observed on premise of aggregation of data, scalability, QoS [2]. 
Fig. 1:- Demonstrate structure for remote sensor hub.

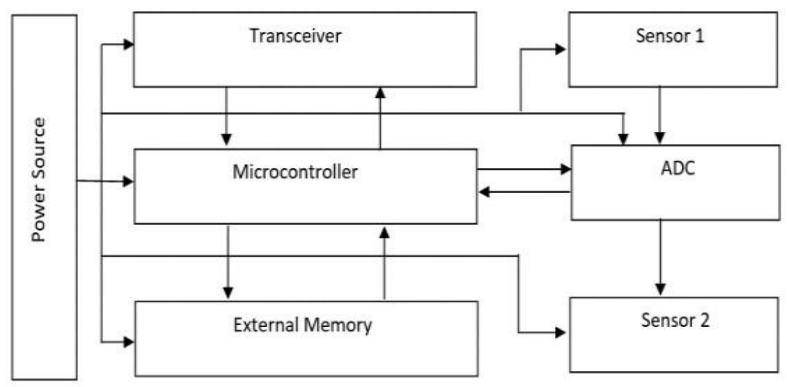

Fig. 1:- Structure for Sensor Node.

\section{Protocol Stack For WSN: -}

The stack of protocol for remote sensor network is based on OSI model that contains seven consecutive layers and on these seven layers five consecutive layers have been chose. These are: physical layer, data link layer, network layer, transport layer, and application layer [1], [3]. The sensor query and data dissemination Protocol (SQDP) gives client applications to send inquiries on the reaction to different inquiries and also gather replies from the inquiries. In this, the last and top most layer i.e., application layer performs different system applications, synchronization of time which incorporates an assortment of used layer protocols. For instance: The SMP (Sensor Management Protocol) is generally an application layer based protocol which gives programming procedures for accomplishment of different assignments. Next the second last layer which is transport layer that accomplishes end-to-end information conveyance from each and every sensor hubs to the nearest sink transport protocol can't stay connected specifically to WSN because of energy and calculation limitation of sensor hubs. It is responsible for catching and storage [14]. When all is done, source hub transmits detected information to sink by means of routing techniques: First routing technique is single hop as well as second on is multi-hop routing technique. In single-hop, main source hub send detected information straightforwardly to sink node which is wide-distant correspondence. In multi-hop routing technique, source hub send detected information to the nearby sink by means of central hubs and is littledistant remote correspondence. In this data delivery contains two streams: upstream and downstream, in upstream the sensor hubs transmit detected information to sink and in downstream information begin from sink, for example, inquiries. The network layer does routing and sends detected information from sensor hubs to sink, in remote sensor organize sensor hubs are conveyed in detecting territory. The data link layer protocol accomplishes information stream multiplexing, information outline development and identification, fault control to give dependable transmission. medium access control (MAC) is imperative capacity of information connection and the goal of MAC is to productively distribute link between different sensor hubs to accomplish great execution on the basis of power utilization, inertness. One more vital capacity is to regulate fault or error and it also comprises two more systems: FEC (Forward Error Correction) accomplishes connect unwavering quality utilizing blunder control in transmission and automatic repeat request (ARQ) ask for accomplishes dependable information broadcast by recommunicating missing information parcels.

The physical layer accomplishes transformation of bit streams to signs from information interface layer that are appropriate for information broadcast across frequency. In physical layer, communication channel and recurrence determination is an imperative issue aimed at correspondence among sensor hubs. MSI (medical, scientific and industrial) and Radio band are choices utilized for communication channel. Consequently, source and beneficiary together speak with each other inside sight range. Protocol stack plays a big role on energy utilization and system lifespan of WSN.

\section{Design Objectives: -}

Network Architecture: -

WSN is made up of three parts - base station or sink, sensor hubs and sensor region. The sensor hubs must automatically sort out themselves into correspondence network and their nature should be self-configurable. Routing is a big challenge and route dependability is critical in part of transfer. speed energy. 


\section{Reliable Environment:-}

The topology of sensor system changes every now and again due to hub catastrophe so hubs ought to be dependable in nature. sensor hubs are connected by remote surrounding which are tends to cause errors.

\section{Energy Capacity: -}

Sensors hubs are controlled by battery and take constrained energy. To draw out the system lifetime energy proficiency ought to be treated as vital viewpoint.

\section{Node Capabilities:-}

Although organization of sensor hubs, hubs ought to be homogenous in nature i.e. have risen to calculation, power limit and correspondence.

Various Routing Protocols in Wireless Sensor Network: -

Table I: - Categorization of Routing Protocols in WSNs

\begin{tabular}{|l|l|}
\hline Information Driven Protocol & SPIN, Directed Diffusion \\
\hline Mobility- based protocols & TTDD, SEAD \\
\hline Location- Aided Protocols & GAF, GEAR, MECN, SMECN \\
\hline In-Network and Layered Processing protocols & LEACH, TEEN, PEGASIS, APTEEN \\
\hline
\end{tabular}

\section{Information Driven Protocol:-}

Information driven protocols, sink node sends a query for gathering information and sensor hubs reacts to question by specifically sending detected information to sink and these are done in traditional routing protocols. In any case, in information driven protocols, source sensor sends information to sink by in-between hubs and likewise accomplish collection of data. Along these lines, information driven routing protocols stores energy by decreasing the transmissions.

\section{SPIN (Sensor Protocols for Information via Negotiation):-}

The SPIN enhances typical flooding issue and this protocol is source alert, source adaptive. SPIN protocol depends on two systems conciliation in this sensor consults with each other before dispersal of any information happens and source adjustment - for energy sparing. SPIN is made of two messages: request (REQ) and advertisement (ADV). In advertisement messages, sensor publicizes its information by sending ADV message containing information that it needs to impart to different sensors. In ask for message, if a sensor inspired by publicized information then it send back a Request message to source of Advertisement that it needs to obtain unique data. Though, SPIN protocols categorised in few sorts: SPIN-BC, SPIN-1 or SPINPP, SPIN-RL, SPIN-EC or SPIN-2.

\section{DD (Directed Diffusion):-}

DD is information driven routing protocol used in inquiry dispersal and handling of inquiry. for example, sensor inside area A may network to choose the best approximation of route of vehicle movement. In this instrument, sensor names their produced information by trait esteem sets and if sensor needs to get information, it sends interests for named information. DD contains key components: information naming, interest, inclination and information reinforcement. One or more small number of paths is reinforces by the sensor network protocol. Sometimes it leads to path failure [10].

\section{Mobility- Based Protocols: -}

The presence of path in remote sensor system is of two sorts: static or portable. In static system, sensors and sink stay in settled position with no development subsequently, have a lesser overhead. In portable system, sensors and sink are in moving position and in this manner, take all the additional overhead however versatile system decreases number of transmissions sparing energy.

\section{TTDD (Two Tier Data Dissemination): -}

Two tier data dissemination protocol was proposed to remove flooding that happens in directed diffusion protocol and furthermore, Two tier data dissemination gives versatile, productive information conveyance. In TTDD, sensors know about their areas however sink could possibly know about its area. TTDD utilizes two instruments: upstream and downstream scattering's protocol has three fundamental stages: framework development, two-level query information sending and framework support. 
SEAD (Scalable Energy-Efficient Asynchronous Dissemination): -

Scalable energy-efficient asynchronous dissemination protocol enhances execution of TTDD protocol and also exchange among limiting the sending delay and saving energy. SEAD accomplishes information dispersal in which source sensor send its detected information to numerous portable sinks and SEAD hold three parts: development of information scattering and uphold linkage to large number of portable sinks.

\section{Location- Aided Protocols:-}

The location data based protocols can be small or extensive area, energy expended relies upon separation over which information is transmitted. Location based protocols utilize area data to guide course revelation and information sending with legitimate energy utilization [1], [4].

\section{GAF (Geographic Adaptive Fidelity): -}

Geographic adaptive fidelity is area based routing protocol proposed for MANETs additionally appropriate for WSNs, the principle component on which geographic adaptive fidelity is based i.e. killing pointless sensors, radio ought to be stopped to give upgrade energy utilization. GAF partitions a sensor field in the form of grid and each sensor hub utilizes area data gave by The Global Positioning System. Three conditions of GAF instrument as appeared in Figure 2: resting state-kill radio when not being used, disclosure state-sensor trade disclosure messages to think about different sensors and in dynamic express a sensor occasionally communicate his exposed messages. The GAF creates robustness [09].

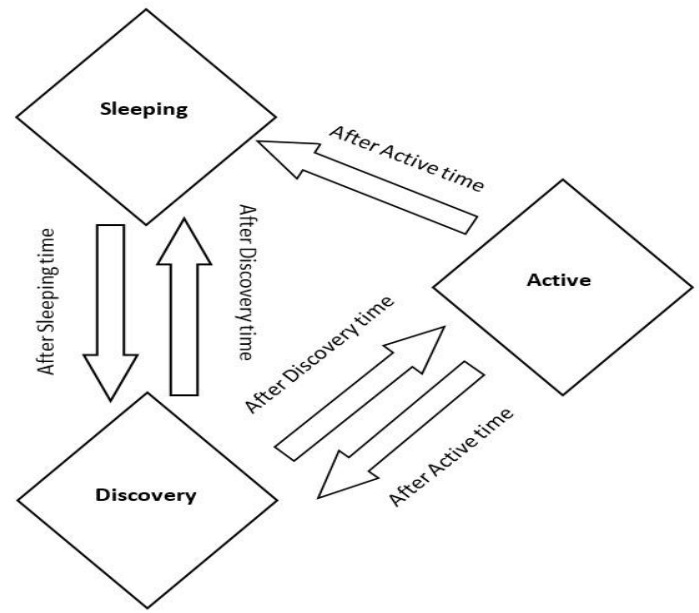

Fig. 2:- Diagram of GAF.

\section{GEAR (Geographic And Energy Aware Routing): -}

GEAR is a energy effective routing convention and proposed to route requests to targeted areas in sensor field. In Adapt additionally, GPS area equipment is furnished by framework to know current positions GEAR utilizes vitality mindful routing based on topographical data to choose sensors to highway a parcel to goal. In this protocol, every sensor keeps an evaluated cost that relies on upon vitality expended and learning taken a toll. Adapt has two stages: sending a bundle towards its goal and spreading the bundle inside goal district. Outfit likewise utilizes confined flooding which is more energy effective in attribute.

\section{MECN (Minimum Energy Communication Network): -}

MECN endeavours to establishment and keep up least energy connect with portable sensors. The fundamental goal of MECN is meant to expand battery lifetime of system and limiting the energy utilization. Likewise, MECN is self-reconfiguring protocol that keeps up system connectivity itself and can powerfully adjust to hub disappointment or including new sensors. Two fundamental periods of MECN: enclosure chart development builds an inadequate diagram, chooses a transfer area (hand-off locale is range where transferring messages to any point through a transitional hub is additional energy productive) and cost dissemination connects they are not ideal are just disposed of. 
SMECN (Small minimum energy communication network): -

SMECN develop negligible chart as for least energy property and develop a system tinier than MECN. While in SMECN sub diagram is developed and each sensor finds its prompt neighbor by communicating revelation messages utilizing beginning force and checks whether subset is set of sensors that answered to disclosure message generally augment energy to re-transmit disclosure message.

\section{Layered And In-Network Processing Protocols: -}

In layered and in-system processing protocol, bunching is done and grouping is a energy proficient correspondence convention utilized through sensors to send detected information to sink. A few groups remain shaped in system with group heads and bunch head is in charge of transmitting detected information beginning from sensors to sink In layered protocol,

\section{LEACH (Low Energy Adaptive Clustering Hierarchy): -}

Low energy adaptive clustering hierarchy is traditional and foremost clustering constructed routing protocol. LEACH utilizes information total strategy that consolidates unique information into littler size of information expelling excess information. LEACH is partitioned into a few groups and is built utilizing limited coordination. It likewise transmits information over short scope of correspondence in this manner decreasing amount of transmissions. The instrument of LEACH is separated into two stages: set up stage and steady stage. In set-up stage, network is sorted out into a few clusters and in steady stage, information transmission to sink is finished. In this protocol, cluster head choice depends on likelihood and sensor choose to end up cluster in light of required rate, current round and set of sensors that have not ended up cluster head in last 1/p round. Along these lines, LEACH is hybrid way utilizing short or long range correspondence and disadvantage of LEACH is that hubs die rapidly [5]. To reduce the amount of data, network is divided into several clusters which is transmitted to the sink [11], [12], [13].

\section{PEAGSIS (Power- Efficient Gathering In Sensor Information System): -}

For enhancement of LEACH performance, PEAGSIS was developed that permit just a single bunch go to transmit information to soak in each round. In this protocol, sensors are composed in chain frame which can be performed by sensor itself or by sink. The development period of this system expect that all sensors have worldwide learning about system and in information combination stage just a single pioneer will transmit information to sink. Subsequently, PEAGSIS has better network than LACH.

\section{TEEN (Threshold sensitive energy efficient sensor network protocol): -}

TEEN is various levelled clustering protocol that objectives a receptive system and in this sensor inside cluster send detected information to cluster head; cluster head additionally send accumulated information to sink. In this component, two edge qualities are transmitted i.e. hard limit and soft limit. Every cluster head in group communicate an incentive to its individuals and that esteem is hard limit for detected quality when achieving hard limit esteem sensor turn on their transmitter to send detected information to sink and furthermore, cluster head communicate another esteem that shows a little change in estimation of detected trait called soft limit. Both hard limit and soft limit lessens the number of transmissions. TEEN is not appropriate for detecting applications which oblige sensors to report information regularly based [15].

\section{APTEEN (Adaptive Periodic TEEN) :-}

There were many disadvantages of TEEN so that APTEEN was designed and APTEEN protocol consolidates elements of both LEACH and TEEN to accomplish better execution. APTEEN is hybrid clustering based routing protocol that permits sensors to send detected information occasionally not on normal premise and give low energy dispersal with bigger number of alive sensors. APTEEN works same as TEEN, after cluster development cluster head communicate edge values. Since, APTEEN can deal with three kind of questions authentic, one time and diligent questions.

\section{Additional Routing Protocols In WSN: - DEEC (Distributed Energy Efficient Clustering Protocol): -}

Distributed energy efficient clustering protocol is clustering or grouping protocol for at least two level heterogeneous system. This cluster head determination is based on leftover energy and normal energy of system. The hubs with higher beginning, leftover energy have more opportunities to come to be cluster head. DEEC is proactive protocol and convey consistent energy [7]. 


\section{HEER (Hybrid Energy-Efficient Reactive Protocol): -}

Hybrid Energy Efficient Reactive is cross breed protocol as it functions admirably both in homogenous and heterogeneous system. HEER is responsive or reactive protocol and does not require any worldwide information of energy when choosing cluster heads. HEER transmit two edge values: hard limit and soft limit. This protocol performs best for time basic applications and diminishes number of transmissions accordingly therefore, economical energy [8].

\section{HEE (Hybrid Energy-Efficient Routing Protocol): -}

Hybrid Energy Efficient was developed to enhance execution of energy utilization when contrasting with through broadcast and least energy broadcast. The Hybrid Energy Efficient protocol proposed is reasonable for both substantial and little size system. HEE is mixture of dualistic straightforward routing methods that is coordinate transmission then least energy transmission. Thusly, HEE gives improved energy proficiency irrespective to system size and message length [6].

\section{Comparisons:-}

Table 2: - Comparison of various routing Protocols in wireless sensor network

\begin{tabular}{|l|l|l|l|}
\hline Various Routing Protocols & Aggregation of information & Scalability & QoS \\
\hline GAF & N & G & N \\
\hline MECN & N & M & N \\
\hline GEAR & N & M & N \\
\hline PEAGSIS & N & G & N \\
\hline SPIN & Y & M & N \\
\hline TTDD & N & G & N \\
\hline LEACH & Y & G & N \\
\hline DEEC & N & M & Y \\
\hline HEER & Y & G & Y \\
\hline TEEN & Y & G & N \\
\hline APTEEN & Y & G & Y \\
\hline
\end{tabular}

Where:

$Y=$ Yes $\quad N=N o \quad G=$ Good $\quad M=$ Medium

\section{Conclusions:-}

Routing in Wireless Sensor Network is innovative zone of research in networking. To give little idleness, energy effective and real-time certifications in sensor system is testing issue however primary concentration is to accomplish better energy utilization. In any case, routing protocol intended for remote sensor system ought to be energy productive and drag out system lifetime. Thus, in this survey paper grouping of routing protocol is sorted into four categories: area based protocols, layered and in system processing protocols, information driven protocols and portability based protocols. Likewise, comparison of routing protocols of remote sensor system is exhibited in this survey paper with respect to QoS and scalability.

\section{References:-}

1. Renna, S. Phulre and R. Pandit, "Wireless Sensor Network and its Routing Protocol: A Survey Paper", 2013.

2. L.A. Villas, D. Guidoni, A. Boukerche, R.B. Araujo and A.F. Loureiro, "Dynamic and Scalable Routing to Perform Efficient Data Aggregation in WSNs", 2011.

3. D. Baghyalakshami, J. Ebenezer and S. A. V. Satyamurty, "Low Latency and Energy efficiemt Routing Protocol for Wireless Sensor network", 2010.

4. J. Zhao and A. T. Erdogan, "A Novel SelfOrganizing Hybrid Network Protocol for wireless sensor network, 2006.

5. L. Tao, Z.Q. Xin and Z. Luqiao, "An Improvement for LEACH Algorithm in Wireless Sensor Network", in Proc. of IEEE 5th Conference on Industrial Electronics and Applications,June 2010.

6. F. Farazandeh, R. Abrishambaf, S. Uyal, T. Gomes and J. Cabral, "A Hybrid Energy-Efficient Routing Protocol for Wireless Sensor Networks, 2013.

7. L. Qing, Q. Zhu and M. Wang, "Design of a distributed energy-efficient clustering algorithm for heterogeneous wireless sensor networks", March 2006. 
8. N. Javaid, S. N. Mohammad, K. Latif, U. Qasim, Z. A. Khan and M. A. Khan, "HEER- Hybrid Energy Efficient Reactive Protocol for Wireless Sensor Networks", 2013.

9. Sinchan Roychowdhury, Chiranjib Patra, Geographic Adaptive Fidelity and Geographic Energy Aware Routing in Ad Hoc Routing,2010.

10. Vandana Jindal, A.K.Verma, Seema Bawa, How the two Adhoc networks can be different:MANET \& WSNs, International Journal of Computer Sci ence \& Technology, Dec. 2011.

11. Geetu, Sonia Juneja, Performance Analysis of SPIN and LEACH Routing Protocol in WSN, International Journal Of Computational Engineering Research (ijceronline.com) Vol. 2 Issue. 5.

12. Rajesh Chaudhary ,Dr. Sonia Vatta, A Tutorial of routing protocols in wireless sensor networks, International Journal of Computer Science and Mobile Computing, June- 2014.

13. Vandana Jindal, A.K.Verma, Seema Bawa, How the two Adhoc networks can be different:MANET \& WSNs, International Journal of Computer Sci ence \& Technology, Dec. 2011.

14. Jennifer Yick, Biswanath Mukherjee, Dipak Ghosal, Wireless sensor network survey, Computer Networks, 2008.

15. Ankita Joshi,Lakshmi Priya M, "A Survey of Hierarchical routing Protocols in Wireless Sensor Networks",MES Journal of Technology and Management. 